\title{
Application of magnetic bead method in detecting coagulation function
}

\author{
Jing Lin, Qixin Li, Weixuan Li
}

Department of Laboratory Medicine, Foshan First People's Hospital, Foshan, China

Contributions: (I) Conception and design: J Lin; (II) Administrative support: W Li; (III) Provision of study materials or patients: J Lin, Q Li; (IV) Collection and assembly of data: Q Li; (V) Data analysis and interpretation: J Lin; (VI) Manuscript writing: All authors; (VII) Final approval of manuscript: All authors.

Correspondence to: Weixuan Li. Department of Laboratory Medicine, Foshan First People's Hospital, 81 Lingnan Street North, Foshan 528000, China. Email: 13902803396@163.com.

\begin{abstract}
Backgroundk Blood samples indicative of jaundice, lipemia, hemolysis, and others are often encountered in the laboratory, and such features impact greatly on the detection of coagulation items. To understand the anti-interference ability of the magnetic bead method automatic coagulation instrument and the optical method automatic coagulation instrument against jaundice, lipemia, and hemolysis, anti-interference experiments of prolonged prothrombin time (PT), activated partial thromboplastin time (APTT), thrombin time (TT), and fibrinogen (FIB) were conducted using ExC810 (magnetic bead method) and CS5100 (optical method).

Methods: Interference samples were prepared with bilirubin, hemoglobin, and lipids, while control samples were prepared with $\mathrm{NaOH}$ and distilled water. The samples contained different values of PT, APTT, TT, and FIB and were detected by magnetic bead method and optical method, respectively. The relative deviation was calculated according to the formula.

Results: In the anti-interference experiment of PT, APTT, TT, and FIB (jaundice, lipemia, hemolysis), the deviation between the test results and the control results with the addition of interfering substances tested by ExC810 was lower overall than that of CS5100. However, after the addition of interfering substances, most of the items were not detected by CS5100 in the anti-lipidemia experiment.

Conclusions: When testing coagulation function, the magnetic bead method has better anti-interference recognition of jaundice, lipemia, and hemolysis than the optical method, and its anti-lipemic interference has a particularly obvious advantage.
\end{abstract}

Keywords: Coagulation function; examination; magnetic bead method; optical method

Submitted Aug 04, 2021. Accepted for publication Oct 22, 2021.

doi: 10.21037/apm-21-2713

View this article at: https://dx.doi.org/10.21037/apm-21-2713

\section{Introduction}

In the laboratory, blood samples are often complicated by presentations such as jaundice, lipemia, hemolysis, and others, and the detection of coagulation items in such samples can be particularly challenging, which could affect the interpretation of the results and diagnosis of diseases by doctors (1-3).

In order to understand the anti-interference ability of the magnetic bead method automatic coagulation instrument and the optical method automatic coagulation instrument against jaundice, lipemia, and hemolysis, an anti-interference experiment of prolonged prothrombin time (PT), activated partial thromboplastin time (APTT), thrombin time (TT), and fibrinogen (FIB) assessment were conducted by Shenzhen Mindray automatic coagulation instrument ExC810 (magnetic bead method; Shenzhen Mindray Bio-Medical Electronics Co., Ltd., Shenzhen, 
Guangdong, China) and Sysmex Automated Coagulation Analyzer CS5100 (optical method; Sysmex Corp., Kobe, Hyogo, Japan), respectively. We present the following article in accordance with the MDAR reporting checklist (available at https://dx.doi.org/10.21037/apm-21-2713).

\section{Methods}

\section{Anti-bilirubin interference experiment}

Preparation of bilirubin interference samples was conducted as follows: mixed plasma samples (more than 20 tubes of coagulated blood samples mixed for preparation) were prepared. One of each abnormal sample (PT, FIB, APTT, TT) was selected of the 4 routine examinations (beyond the normal reference range), respectively. A total of $0.02 \mathrm{~g}$ of bilirubin was weighed and dissolved in $2.5 \mathrm{~mL}$ of $0.1 \mathrm{M}$ $\mathrm{NaOH}$ to prepare a bilirubin mother solution of $800 \mathrm{mg} / \mathrm{dL}$. The ratios of bilirubin mother solution and mixed plasma samples were calculated to mix and prepare 40 and $80 \mathrm{mg} / \mathrm{dL}$ interference sample of PT; 40 and $80 \mathrm{mg} / \mathrm{dL}$ interference sample of APTT; 80 and $100 \mathrm{mg} / \mathrm{dL}$ interference sample of TT; 100 and $160 \mathrm{mg} / \mathrm{dL}$ interference sample of FIB; another mixed plasma sample with $0.1 \mathrm{M} \mathrm{NaOH}$ was taken to correspondingly prepare 40 and $80 \mathrm{mg} / \mathrm{dL}$ control sample of PT; 40 and $80 \mathrm{mg} / \mathrm{dL}$ control sample of APTT; 80 and $100 \mathrm{mg} / \mathrm{dL}$ Control sample of TT; and 100 and $160 \mathrm{mg} / \mathrm{dL}$ control sample of FIB. The ratio of bilirubin mother solution and abnormal plasma sample was calculated, and a PT $40 \mathrm{mg} / \mathrm{dL}$ interference sample was mixed and configured; $40 \mathrm{mg} / \mathrm{dL}$ interference sample of APTT; $80 \mathrm{mg} / \mathrm{dL}$ interference sample of TT; $100 \mathrm{mg} / \mathrm{dL}$ interference sample of FIB; another copy with $0.1 \mathrm{M} \mathrm{NaOH}$ abnormal plasma samples was taken to correspondingly prepare $40 \mathrm{mg} / \mathrm{dL}$ control samples of PT; $40 \mathrm{mg} / \mathrm{dL}$ control samples of APTT; $80 \mathrm{mg} / \mathrm{dL}$ control samples of TT; and $100 \mathrm{mg} / \mathrm{dL}$ control samples of FIB. Each coagulometer was calibrated before the experiment, and the quality control of all test items was passed with the supporting reagents. The test was repeated twice for the samples with 2 concentrations of mixed plasma and the corresponding control samples, mean value of the sample concentration was calculated, abnormal plasma sample and the control sample was tested once, data were recorded in Table 1, and the relative deviation was calculated according to the formula. The formula was as follows: deviation $=[\mathrm{y}$ $-x) / x] \times 100 \%$, where $y$ is the detection value of interference sample, and $x$ is the detection value of control sample (the same as below). All procedures performed in this study involving human participants were in accordance with the Declaration of Helsinki (as revised in 2013). The study was approved by institutional ethics board of Foshan First People's Hospital [No. L(2020)23] and informed consent was taken from all the patients.

\section{Anti-bemoglobin interference experiment}

Preparation of hemoglobin interference samples: mixed plasma samples (preparation of coagulation samples with more than 20 tubes) were prepared. One sample was selected from each abnormal sample type of the 4 routine examinations (PT, FIB, APTT, TT; beyond the normal reference range). The ratio of hemoglobin interferent $(28,400 \mathrm{mg} / \mathrm{dL})$ and mixed plasma sample was calculated, then 3,155 and $3,550 \mathrm{mg} / \mathrm{dL}$ interference sample of PT, APTT, TT, and FIB was prepared with distilled water, respectively; another mixed plasma sample was taken, then 3,155 and 3,550 mg/dL control sample of PT, APTT, TT, and FIB was prepared with distilled water, respectively. The ratio of hemoglobin interferents and abnormal plasma samples was calculated, then $3,155 \mathrm{mg} / \mathrm{dL}$ interference sample of PT, APTT, TT, and FIB was prepared with distilled water, respectively; another abnormal plasma sample was taken, $3,155 \mathrm{mg} / \mathrm{dL}$ control sample of PT, APTT, TT, and FIB was prepared with distilled water, respectively. Each coagulometer was calibrated before the experiment, and quality control of all test items was conducted and passed with the supporting reagents. The test was repeated twice for the samples with 2 concentrations of mixed plasma and the corresponding control samples, mean value of the sample concentration was calculated, abnormal plasma sample and the control sample was tested once, data were recorded in Table 2, and the relative deviation was calculated according to the formula.

\section{Anti-lipid interference experiment}

Preparation of blood lipid interference sample was as follows: a mixed plasma sample (mix preparation of coagulation samples with more than 20 tubes) was prepared. A sample was selected of each abnormal sample of the 4 routine examinations (PT, FIB, APTT, TT; beyond the normal reference range). The ratios with blood lipid interference substances $(50,000 \mathrm{mg} / \mathrm{dL})$ and mixed plasma samples were calculated to mix and prepare as 2,500 and 2,941 mg/dL interference samples of PT; 2,941 
Table 1 Experiment results of anti-bilirubin interference

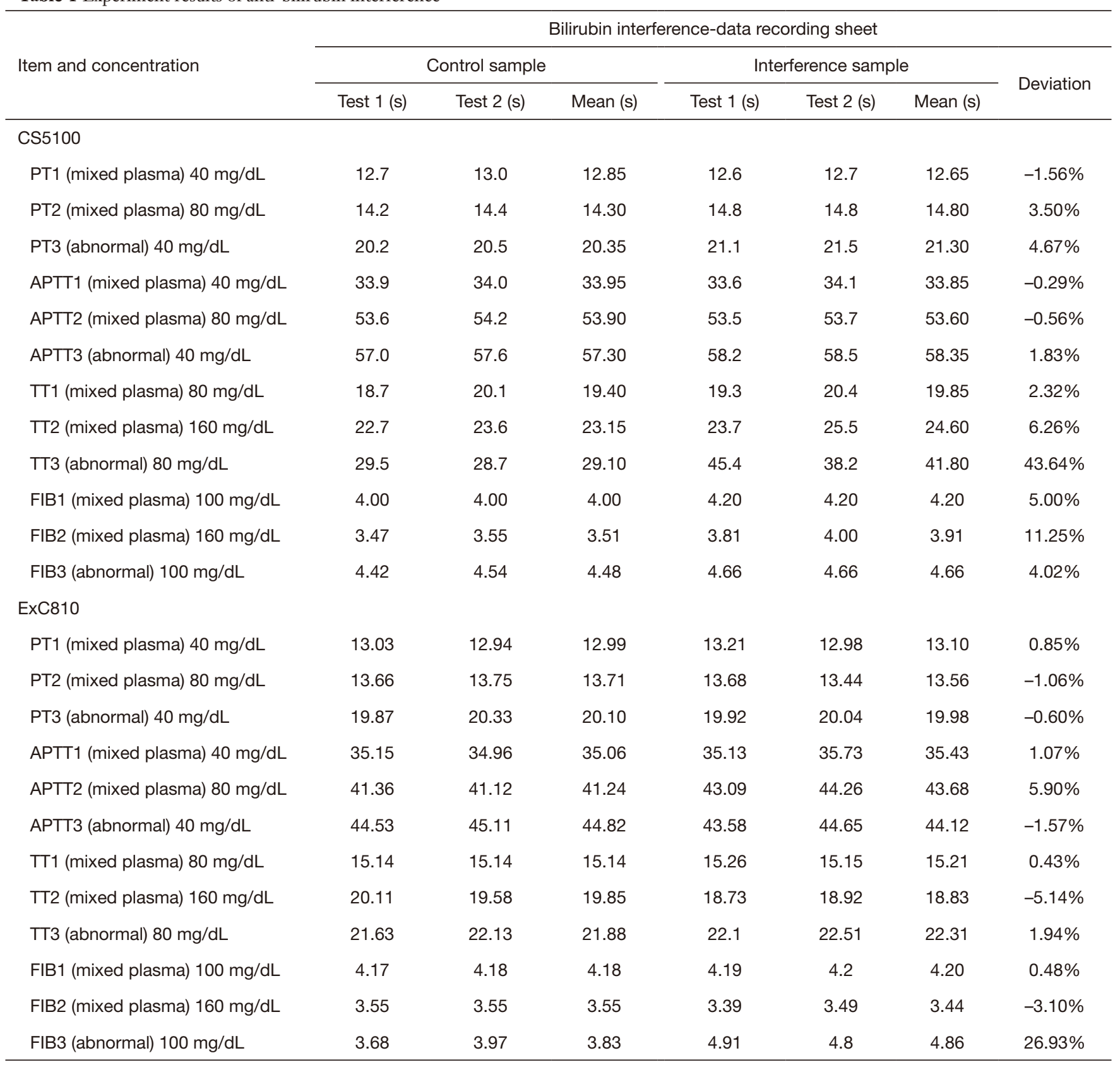

PT, prothrombin time; APTT, activated partial thromboplastin time; TT, thrombin time; FIB, fibrinogen.

and $3,333 \mathrm{mg} / \mathrm{dL}$ interference samples of APTT; 1,666 and $2,000 \mathrm{mg} / \mathrm{dL}$ interference samples of TT; 4,504 and $5,000 \mathrm{mg} / \mathrm{dL}$ interference sample of FIB; another mixed plasma sample was taken with distilled water to correspondingly prepare as 2,500 and 2,941 $\mathrm{mg} / \mathrm{dL}$ control sample of PT; 2,941 and 3,333 $\mathrm{mg} / \mathrm{dL}$ control sample of APTT; 1,666 and 2,000 mg/dL control sample of TT;
4,504 and 5,000 mg/dL control sample of FIB. The ratios of blood lipid interference substances and abnormal plasma samples were calculated to mix and prepare a $2,500 \mathrm{mg} / \mathrm{dL}$ interference sample of PT; 2,941 $\mathrm{mg} / \mathrm{dL}$ interference sample of APTT; $1,666 \mathrm{mg} / \mathrm{dL}$ interference sample of TT; $4,504 \mathrm{mg} / \mathrm{dL}$ interference sample of FIB; another abnormal plasma sample was taken with the distilled water to 
Table 2 Experiment results of anti-hemoglobin interference

\begin{tabular}{|c|c|c|c|c|c|c|c|}
\hline \multirow{2}{*}{ Item and concentration } & \multicolumn{7}{|c|}{ Hemoglobin interference-data recording sheet } \\
\hline & Test 1 (s) & Test 2 (s) & Mean (s) & Test 1 (s) & Test 2 (s) & Mean (s) & Deviation \\
\hline \multicolumn{8}{|l|}{ CS5100 } \\
\hline PT1 (mixed plasma) 3,155 mg/dL & 11.10 & 12.00 & 11.55 & 11.70 & 11.90 & 11.80 & $2.16 \%$ \\
\hline PT3 (abnormal) 3,155 mg/dL & 20.00 & & 20.00 & 20.30 & & 20.30 & $1.50 \%$ \\
\hline APTT1 (mixed plasma) 3,155 mg/dL & 30.30 & 28.20 & 29.25 & 28.30 & 27.70 & 28.00 & $-4.27 \%$ \\
\hline APTT2 (mixed plasma) $3,550 \mathrm{mg} / \mathrm{dL}$ & 30.60 & 29.00 & 29.80 & 29.00 & 29.50 & 29.25 & $-1.85 \%$ \\
\hline APTT3 (abnormal) 3,155 mg/dL & 59.10 & & 59.10 & 44.20 & & 44.20 & $-25.21 \%$ \\
\hline TT3 (abnormal) 3,155 mg/dL & 37.90 & & 37.90 & 53.70 & & 53.70 & $41.69 \%$ \\
\hline FIB1 (mixed plasma) $3,155 \mathrm{mg} / \mathrm{dL}$ & 2.99 & 3.18 & 3.09 & 3.05 & 2.87 & 2.96 & $-4.05 \%$ \\
\hline FIB2 (mixed plasma) $3,550 \mathrm{mg} / \mathrm{dL}$ & 2.99 & 2.93 & 2.96 & 2.99 & 2.71 & 2.85 & $-3.72 \%$ \\
\hline FIB3 (abnormal) 3,155 mg/dL & 4.79 & & 4.79 & 4.10 & & 4.10 & $-14.41 \%$ \\
\hline \multicolumn{8}{|l|}{ ExC810 } \\
\hline PT1 (mixed plasma) 3,155 mg/dL & 12.37 & 12.54 & 12.46 & 12.01 & 12.22 & 12.12 & $-2.73 \%$ \\
\hline PT2 (mixed plasma) 3,550 mg/dL & 12.51 & 12.58 & 12.55 & 11.95 & 11.87 & 11.91 & $-5.06 \%$ \\
\hline PT3 (abnormal) 3,155 mg/dL & 19.52 & & 19.52 & 18.35 & & 18.35 & $-5.99 \%$ \\
\hline TT3 (abnormal) 3,155 mg/dL & 23.89 & & 23.89 & 23.67 & & 23.67 & $-0.92 \%$ \\
\hline FIB1 (mixed plasma) $3,155 \mathrm{mg} / \mathrm{dL}$ & 3.35 & 3.22 & 3.29 & 3.15 & 3.27 & 3.21 & $-2.28 \%$ \\
\hline FIB2 (mixed plasma) $3,550 \mathrm{mg} / \mathrm{dL}$ & 3.22 & 3.25 & 3.24 & 3.31 & 3.17 & 3.24 & $0.15 \%$ \\
\hline FIB3 (abnormal) 3,155 mg/dL & 5.14 & & 5.14 & 5.17 & & 5.17 & $0.58 \%$ \\
\hline
\end{tabular}

PT, prothrombin time; APTT, activated partial thromboplastin time; TT, thrombin time; FIB, fibrinogen.

correspondingly prepare as $2,500 \mathrm{mg} / \mathrm{dL}$ control sample of PT; 2,941 mg/dL control sample of APTT; $1,666 \mathrm{mg} / \mathrm{dL}$ control sample of TT; 4,504 mg/dL control sample of FIB. Each coagulometer was calibrated before the experiment, and quality control of all test items was completed and passed with the supporting reagents. The test was repeated twice for the samples with 2 concentrations of mixed plasma and the corresponding control samples, the mean value of the sample concentration was calculated, abnormal plasma sample and the control sample was tested once, data were recorded in the following Table 3, and the formula was used to calculate relative deviation. 
Table 3 Experiment results of anti-lipid interference

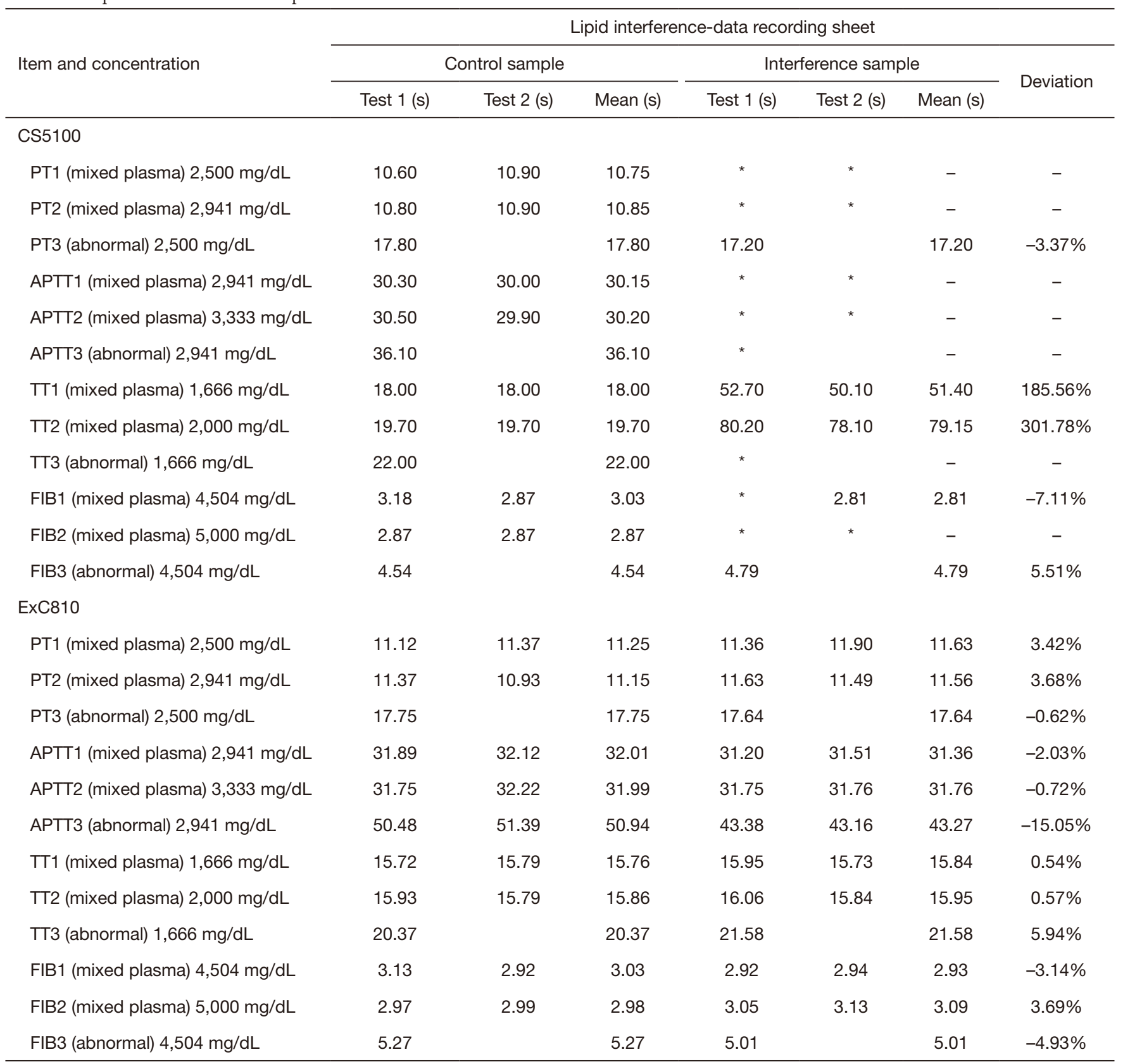

${ }^{*}$, the undetectable. -, no data measured. PT, prothrombin time; APTT, activated partial thromboplastin time; TT, thrombin time; FIB, fibrinogen.

\section{Statistical methods}

Calculation of interference result: calculate the offset value according to EP7-A2 file, and the offset value $=\overline{\mathrm{X}}$ test $-\bar{X}$ control. Observe the interference.

\section{Results}

\section{Anti-bilirubin interference experiment}

In the experiment, when bilirubin was added for interference, the deviation on the detection results of 


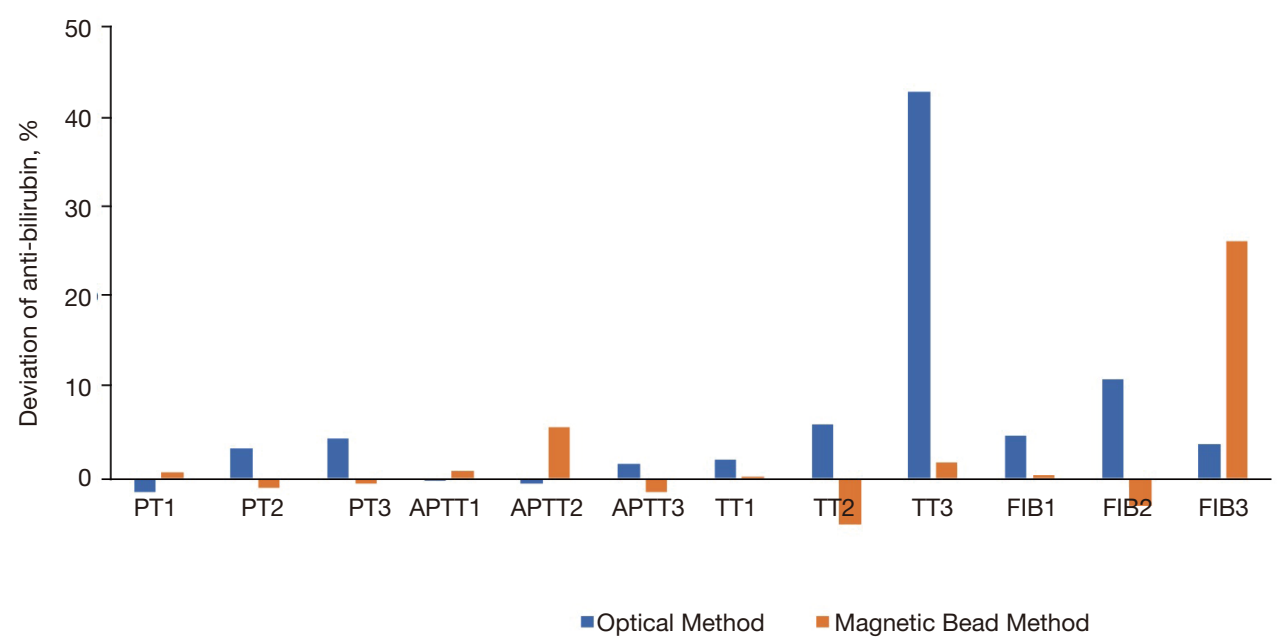

Figure 1 Deviation of anti-bilirubin. PT, prothrombin time; APTT, activated partial thromboplastin time; TT, thrombin time; FIB, fibrinogen.

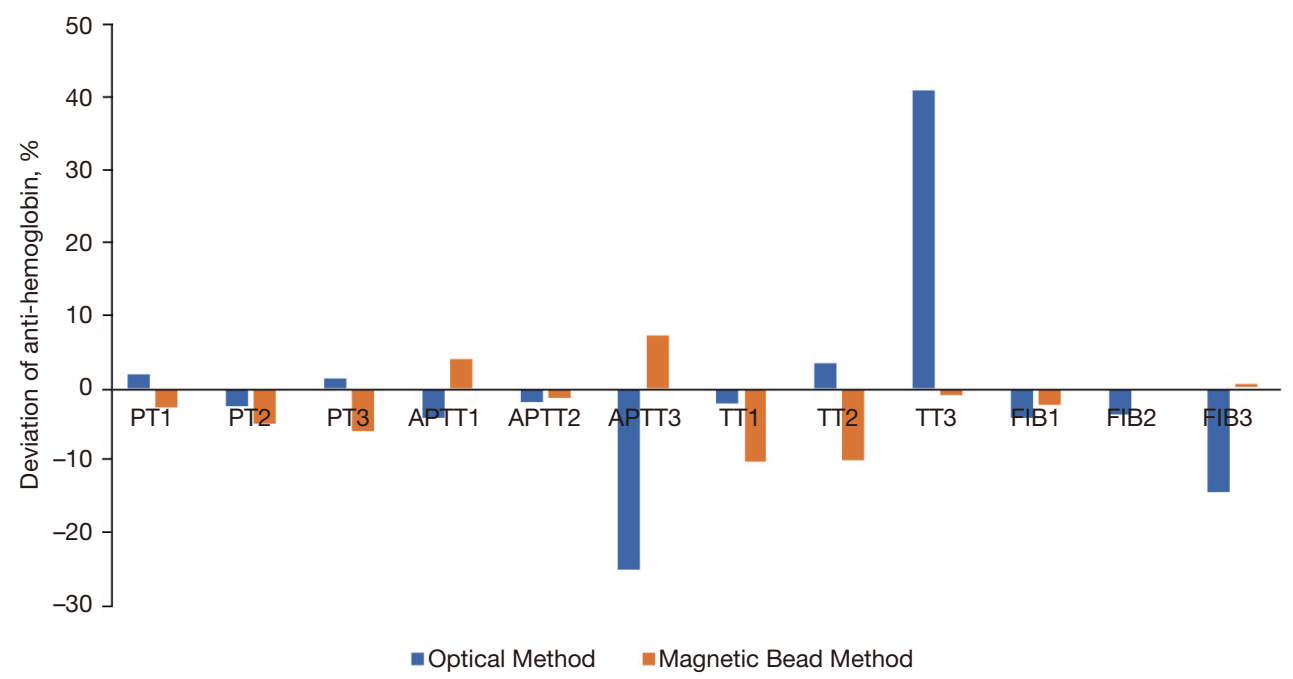

Figure 2 Deviation of anti-hemoglobin. PT, prothrombin time; APTT, activated partial thromboplastin time; TT, thrombin time; FIB, fibrinogen.

the optical method was generally greater than that of the magnetic bead method, especially the detection results of PT and TT, as shown in Table 1 and Figure 1.

\section{Anti-bemoglobin interference experiment}

In the experiment, when hemoglobin was added for interference, the overall deviation of the optical method was greater than that of the magnetic bead method, especially the detection results of PT, APTT, and FIB, as shown in Table 2 and Figure 2.

\section{Anti-lipid interference experiment results}

In the experiment, when lipids were added for interference, the overall deviation of the optical method detection result was greater than that of the magnetic bead method, especially the optical method often failed to detect the 


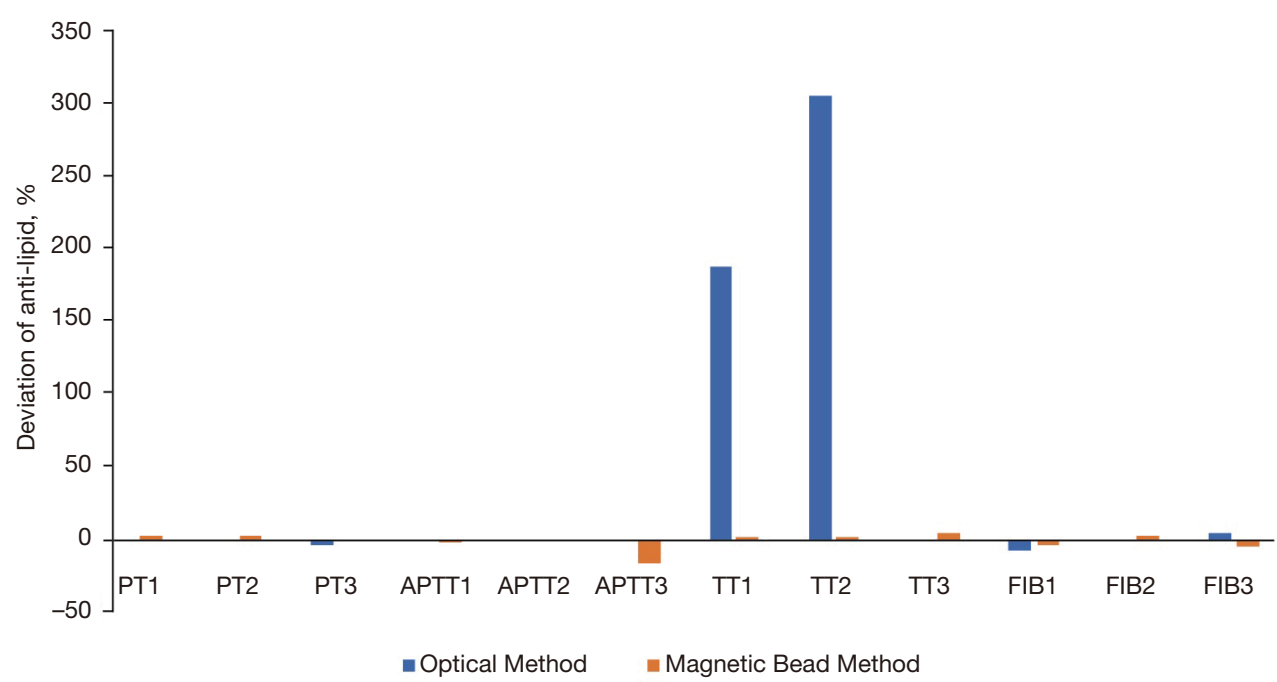

Figure 3 Deviation of anti-lipid. PT, prothrombin time; APTT, activated partial thromboplastin time; TT, thrombin time; FIB, fibrinogen.

specific values, as shown in Table 3 and Figure 3 (no tested results were presented as the missing part during drawing).

\section{Discussion}

In the anti-interference experiment of PT, APTT, TT, and FIB, after adding interfering substances (bilirubin, hemoglobin, and blood lipid), the overall deviation of the test results and the control results of $\mathrm{ExC} 810$ was lower than that of CS5100. However, most of the items failed detection in the results after adding interfering substances in the anti-lipidemia experiment of CS5100. Therefore, based on the current results, it appears that magnetic bead method instruments (such as ExC810) are better than optical instruments (such as CS5100) in anti-jaundice, lipemia, and hemolysis interference and the advantage of anti-lipemia interference is particularly significant.

Currently, there are 2 methods for detecting blood coagulation function - the optical method and magnetic bead method, among which the optical method is easily affected by blood components. In some disease states, blood pressure components often increase abnormally, and these abnormalities often combine with changes in coagulation function. It is more common in clinical practice that bilirubin is significantly increased with abnormal liver function, and coagulation function is decreased in severe cases, while the optical method is susceptible to the influence of bilirubin increase, resulting in inaccurate coagulation function test results. For the same reason, anticoagulation is often required for patients with acute coronary syndrome, whether it is in interventional surgery or the acute phase, and many of these patients have hyperlipidemia, which may also affect the test results of coagulation function. In recent years, the magnetic bead method has been widely used and subjected to in-depth research in sample detection $(4,5)$. While the magnetic bead method mainly considers viscosity changes, the changes in mechanical motion are detected rather than optical changes. Therefore, it is not affected by jaundice, lipids, high concentrations of hemoglobin, and turbidity. The results of the study basically confirmed this viewpoint. In most of the test results, the deviation of the magnetic bead method was lower than that of the optical method, the latter was particularly susceptible to interferences in the detection of TT, it is often impossible to measure the result of TT with the lipid interference especially, and the magnetic bead method showed a better anti-interference ability. Therefore, the magnetic bead method can be preferred in the determination of coagulation function for patients who may have interferences such as the aforementioned jaundice, lipids, hemoglobin, and others.

However, there are some problems in the practical use of the magnetic bead method that should be taken into account. We know that temperature has a certain effect on coagulation function (6). It has been found that the accuracy of the magnetic bead method to detect coagulation function is significantly affected by temperature, and there are some differences in the coagulation function measured 
at different temperatures (7). Therefore, the investigators suggested that when coagulation function is detected by the magnetic bead method, the indoor quality control should be established according to different temperatures (8). Secondly, the coagulation function analyzer used for the magnetic bead method has a complicated structure, for which the obtained single value of coagulation endpoint is not conducive to subsequent research. Integrating the results of numerous related studies, it appears that the optimum blood clotting time for magnetic bead detection is $8-25 \mathrm{~s}$. As the clotting factors in platelets will affect the measurement results, the plasma must be platelet-poor; dilution with buffer plasma must be accurate, so that the optimum clotting time is $8-25 \mathrm{~s}$, which is beneficial to FIB detection; reagents must be prepared and used immediately, otherwise the activity of thrombin is likely to be insufficient or disappear, and the clotting time will be prolonged; each laboratory must establish its own standard curve and monitor it closely. If there is any change of conditions, the standard should be re-established.

This study had some limitations. The simulated samples were adapted in the study without verification for performing the comparative tests of patients in clinical practices; moreover, the effects of temperature were not further verified. Future studies should include specific patients (such as patients with abnormally elevated bilirubin, abnormally elevated or decreased hemoglobin, and significantly elevated blood lipids) for comparative studies with the gold standard.

\section{Acknowledgments}

Funding: None.

\section{Footnote}

Reporting Checklist: The authors have completed the MDAR reporting checklist. Available at https://dx.doi. org/10.21037/apm-21-2713

Data Sharing Statement: Available at https://dx.doi. org/10.21037/apm-21-2713

Conflicts of Interest: All authors have completed the ICMJE uniform disclosure form (available at https://dx.doi. org/10.21037/apm-21-2713). The authors have no conflicts of interest to declare.
Ethical Statement: The authors are accountable for all aspects of the work in ensuring that questions related to the accuracy or integrity of any part of the work are appropriately investigated and resolved. All procedures performed in this study involving human participants were in accordance with the Declaration of Helsinki (as revised in 2013). The study was approved by institutional ethics board of Foshan First People's Hospital (No. L(2020)23) and informed consent was taken from all the patients.

Open Access Statement: This is an Open Access article distributed in accordance with the Creative Commons Attribution-NonCommercial-NoDerivs 4.0 International License (CC BY-NC-ND 4.0), which permits the noncommercial replication and distribution of the article with the strict proviso that no changes or edits are made and the original work is properly cited (including links to both the formal publication through the relevant DOI and the license). See: https://creativecommons.org/licenses/by-nc-nd/4.0/.

\section{References}

1. Cakir T, Cingi A, Yeğen C. Coagulation dynamics and platelet functions in obstructive jaundiced patients. J Gastroenterol Hepatol 2009;24:748-51.

2. Negrini D, Bernardi D, Antonelli G, et al. Interference of lipemia in samples for routine coagulation testing using a Sysmex CS-5100 coagulometer. Int J Lab Hematol 2019;41:772-7.

3. Nougier C, Jousselme E, Sobas F, et al. Effects of hemolysis, bilirubin, and lipemia interference on coagulation tests detected by two analytical systems. Int J Lab Hematol 2020;42:88-94.

4. Andrews WT, Skube SB, Hummon AB. Magnetic beadbased peptide extraction methodology for tissue imaging. Analyst 2017;143:133-40.

5. Jalal UM, Jin GJ, Eom KS, et al. On-chip signal amplification of magnetic bead-based immunoassay by aviating magnetic bead chains. Bioelectrochemistry 2018;122:221-6.

6. Wolberg AS, Meng ZH, Monroe DM 3rd, et al. A systematic evaluation of the effect of temperature on coagulation enzyme activity and platelet function. $\mathrm{J}$ Trauma 2004;56:1221-8.

7. Yazar H, Özdemir F, Köse E. Effect of Centrifuge Temperature on Routine Coagulation Tests. Acta Haematol 2018;139:158-63. 
8. Kristoffersen AH, Stavelin AV, Ajzner E, et al. Preanalytical practices for routine coagulation tests in European laboratories. A collaborative study from the European Organisation for External Quality Assurance
Providers in Laboratory Medicine (EQALM). Clin Chem Lab Med 2019;57:1511-21.

(English Language Editor: J. Jones)

Cite this article as: Lin J, Li Q, Li W. Application of magnetic bead method in detecting coagulation function. Ann Palliat Med 2021;10(10):10607-10615. doi: 10.21037/apm-21-2713 